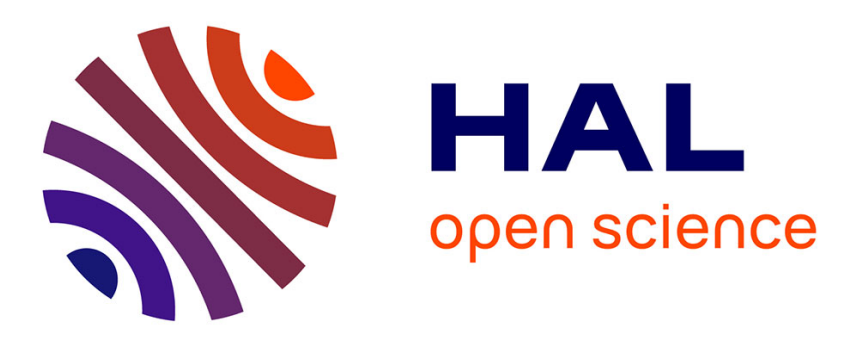

\title{
A symbolic approach for the identification of radiative properties
}

M. Galtier, Maxime Roger, Frédéric André, Agnès Andrée Delmas

\section{To cite this version:}

M. Galtier, Maxime Roger, Frédéric André, Agnès Andrée Delmas. A symbolic approach for the identification of radiative properties. Journal of Quantitative Spectroscopy and Radiative Transfer, 2017, 196, pp.130-141. 10.1016/j.jqsrt.2017.03.026 . hal-01871396

\section{HAL Id: hal-01871396 https://hal.science/hal-01871396}

Submitted on 26 Oct 2018

HAL is a multi-disciplinary open access archive for the deposit and dissemination of scientific research documents, whether they are published or not. The documents may come from teaching and research institutions in France or abroad, or from public or private research centers.
L'archive ouverte pluridisciplinaire HAL, est destinée au dépôt et à la diffusion de documents scientifiques de niveau recherche, publiés ou non, émanant des établissements d'enseignement et de recherche français ou étrangers, des laboratoires publics ou privés. 


\title{
A symbolic approach for the identification of radiative properties
}

\author{
M. Galtier ${ }^{\mathrm{a}}$, M. Roger ${ }^{\mathrm{a}, *}$, F. Andréa ${ }^{\mathrm{a}}$ A. Delmas ${ }^{\mathrm{a}}$ \\ ${ }^{a}$ Univ Lyon, CNRS, INSA-Lyon, Université Claude Bernard Lyon 1, CETHIL UMR5008, F-69621, \\ Villeurbanne, France
}

\begin{abstract}
A new Symbolic Monte Carlo (SMC) based on null-collision algorithms, allows 1/ overcoming the usually required knowledge of the optical thickness in SMC, and 2/ expressing radiative quantities as simple polynomials of the absorption and scattering coefficients. The proposed method can be applied to complex systems such as heterogeneous absorbing and scattering media in complex geometry. It opens new outlooks for the analysis and the identification of radiative properties in a wide range of radiative transfer applications in participating media.
\end{abstract}

Keywords: Symbolic Monte Carlo (SMC), Null collisions, Polynomials, Radiative properties, Identification

\section{Introduction}

The difference between Symbolic Monte Carlo (SMC) methods and standard Monte Carlo (MC) methods lies in the fact that some parameters are retained as symbolic variables in SMC, while scalar values are affected to all the problem's parameters in MC. Consequently, the output of SMC simulations is a functional in which the requested parameters appear explicitly. The symbolic approach thus differs from other more usual techniques, such as standard MC, for which scalar numerical outputs are obtained.

The SMC functional form is generally used to infer the parameters of interest, appearing as variables in the function, from measurements. Indeed, SMC makes the resolution of inverse problems easier, which may explain why it was initially called inverse Monte Carlo method [1]. SMC methods have been applied in various studies

*Corresponding author. Tel.: +33472 438251

Email address: maxime.roger@insa-lyon.fr (M. Roger) 
dedicated to inverse problems in remote sensing [2] or medical imaging [3, 4]. In particular, SMC have been investigated by Dunn [5] and by Subramaniam and Mengüç [6] for inverse problems in radiative heat transfer.

It should be emphasized that SMC methods only solve the direct model, and are completely distinct from usual MC techniques developed for solving inverse problems such as Markov Chain Monte Carlo methods within the Bayesian framework [1, 7]. The symbolic approach can be considered as a useful preliminar step toward the development of an efficient identification strategy. In radiative transfer problems, these identification methods combine spectroscopic measurements with radiation models, such as the radiative transfer equation (RTE), in order to infer parameters such as the radiative properties, temperature or species concentrations [8]. Inverse problems for radiation concern many applications including optical tomography in medical imaging [9] or combustion diagnosis [10] among others. Inverse radiation methods have also been implemented for the identification of radiative properties in complex heterogeneous media such as porous and fibrous materials [11, 12, 13, 14, 15], or foams $[16,17]$. In these approaches, the inversion is performed by using an iterative procedure, where at each step, direct computations and measurements (of radiative fluxes, intensities) are compared.

The objective of this work is to propose a SMC algorithm that allows obtaining simple analytical forms (here polynomials) of radiative quantities as a function of radiative properties, and that can be applied in complex problems related to radiative transfer (in complex three-dimensional geometry, with absorption, scattering and/or reflective walls, etc.). The analytical form can then be used $1 /$ to help in the choice of an inverse strategy by providing physical insights about the parameters which need to be identified, and $2 /$ to improve the efficiency of the inverse method. For instance, if a minimization technique is applied, the analytical forms of the radiative quantities are used as solution of the direct model. Consequently, the numerical resolution of the direct model is not needed anymore at each iteration which significantly decreases the computational cost. This is particularly true in many radiative transfer applications where the numerical resolution of the radiative transfer equation (RTE), commonly used as direct radiation model, is computationally expensive [8].

Using SMC, Dunn [5] identified the scattering albedo in inhomogeneous media assuming isotropic scattering. Subramaniam and Mengüç [6] extended the approach 
to anisotropically scattering media, and identified the profile of the scattering albedo together with the asymmetry factor of the phase function. In these studies, the analysis is limited as it requires a precise knowledge of the optical thickness of the medium, making impossible the identification of absorption and scattering coefficients.

In the present work, a new symbolic approach is described that allows expressing radiative quantities (intensities, fluxes, source terms, etc.) as simple functions of the radiative properties of the medium (more specifically its absorption and scattering coefficients). This approach does not require any knowledge about the optical thickness. It opens new outlooks for the development of identification techniques of the radiative properties of participating media since the absorption and scattering coefficients can then be identified, which was not the case for previous SMC studies for which only the albedo was accessible.

The paper is structured as follows. In the second section, the principle of the method is introduced and the potential benefits for modeling and inverse analysis are highlighted. In the third and fourth sections, details of the proposed approach are given. It is shown to provide tractable multivariate polynomials of absorption and scattering coefficients. The developments focus on the absorption and scattering coefficients. The symbolic approach for the phase function parameters has already been discussed by Subramaniam and Mengüç [6], and their approach can be integrated without additional difficulties in the method proposed here. In the fifth section, an example of application in a heterogeneous medium is provided.

\section{Principle}

The objective of the present work is to propose a method for expressing radiative quantities as simple functions of absorption and scattering coefficients of complex participating media. Such functions present advantages in terms of analysis - for a better understanding of physical phenomena, experimentation design or optimization issues - and allow performing inversion procedures with efficiency. Fig. 1, for instance, displays the functional expression of radiative intensity $I_{\eta}$ (at the exit of a homogeneous emitting, absorbing, and isotropically scattering slab of width $L$ ) as a function of the scattering optical thickness $\tau_{s, \eta}=\sigma_{\eta} L$.

The interest of expressing $I_{\eta}$ as a functional is multiple. From a measured value of the radiative intensity, it is easy to identify graphically or analytically the scatter- 


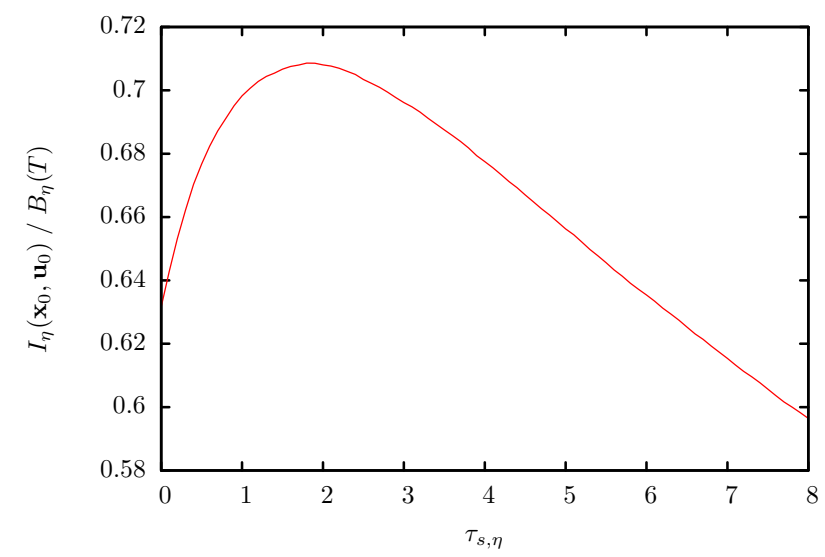

Figure 1: Radiative intensity $I_{\eta}\left(\mathbf{x}_{0}, \mathbf{u}_{0}\right) / B_{\eta}(T)$ versus the scattering optical thickness $\tau_{s, \eta} . \mathbf{x}_{0}$ is located at the exit of the slab, and $\mathbf{u}_{0}$ is the outgoing normal at $\mathbf{x}_{0}$. The absorption optical thickness is $\tau_{a, \eta}=\kappa_{\eta} L=1$ and the medium is isothermal. $B_{\eta}(T)$ is the blackbody intensity at temperature $T$ of the medium.

ing coefficient. Moreover, this figure provides another relevant information: the range of scattering optical thickness for which the identification is likely to be accurate or not. For $\tau_{s, \eta}<1$ and $\tau_{s, \eta}>3$, the radiative intensity depends significantly on $\tau_{s, \eta}$. Therefore, the identification of the scattering coefficient can be implemented with confidence if a priori information indicates that the propagation medium is optically thin or thick. If no a priori information on the propagation medium is available, the information about the uniqueness of the solution is provided by the functional. Indeed, if the value of $I_{\eta}\left(\mathbf{x}_{0}, \mathbf{u}_{0}\right) / B_{\eta}(T)$ derived from measurements is for instance 0.68, two scattering optical thicknesses ( $\tau_{s, \eta}$ may be close to 0.5 or close to 4 ) are solutions of the problem. For intermediate scattering optical thicknesses, i.e. $\tau_{s, \eta} \in[1,3]$, the sensitivity of the radiative intensity to the scattering coefficient is weaker which has strong consequences for inversion. For instance, if the measured value of $I_{\eta}\left(\mathbf{x}_{0}, \mathbf{u}_{0}\right) / B_{\eta}(T)$ is around 0.7 with an uncertainty of 0.02 (relative uncertainty of $3 \%$ ), it is impossible to conclude about which $\tau_{s, \eta}$ is solution in the interval $[0.5,4]$.

All the elements of analysis given by the functional expression are in fact valuable to develop identification strategies.

Functional forms of radiative quantities can be obtained with SMC as shown in [5, 6]. This method is based on the same principle as standard Monte Carlo methods. The only difference is that parameters (such as the absorption and scattering coefficients in our context) are retained under a symbolic form and are not used as "simple" numbers 
(see chapter 7 from Ref. [1]). The quantities evaluated are therefore functions of the retained parameters, while in standard MC, scalars are computed according to a given set of parameter values. In practice, both algorithms are strictly equivalent: a large number of optical paths are randomly generated according to the statistical laws of radiative transfer, and radiative intensities or fluxes are then estimated from the average of samples, under their scalar (in standard MC) or functional forms (in SMC).

Among the statistical laws of radiative transfer in participating media, Beer's law turns out to be critical in SMC for radiative properties identification. Indeed, Beer's law is an exponential of the optical thickness depending on the absorption and scattering coefficients. Therefore, as far as absorption and scattering coefficients are involved, the functional form obtained with SMC remains a hardly tractable average of exponential functions (composed of as many nonlinear terms as Monte Carlo samples). It justifies probably why SMC has not been applied in the last two decades for inverse problems in radiative transfer.

However, in some cases, tractable functions of the absorption and scattering coefficients may be derived from radiative transfer models. For instance, let us consider the radiative transfer equation in the simple case of a non-scattering homogeneous medium

$$
\frac{d I_{\eta}}{d s}=-\kappa_{\eta} I_{\eta}+\kappa_{\eta} B_{\eta}
$$

and assume that there is no radiative source at location $s_{0}$. The radiative intensity at location $s_{j}=s_{0}+j \Delta s$ can be approximated, using finite differences with constant mesh size $\Delta s$, as:

$$
I_{\eta}\left(s_{j}\right)=\kappa_{\eta} \Delta s B_{\eta}+\kappa_{\eta} \Delta s\left(1-\kappa_{\eta} \Delta s\right) B_{\eta}+\cdots+\kappa_{\eta} \Delta s\left(1-\kappa_{\eta} \Delta s\right)^{j-1} B_{\eta}
$$

This equation provides a polynomial function of the absorption coefficient and can be rewritten in a more compact form as:

$$
I_{\eta}\left(s_{j}\right)=\sum_{i=0}^{j-1} a_{i} \kappa_{\eta}^{i}
$$

Obviously in this simple case, the radiative intensity is known analytically and the polynomial expression has no real interest. Nevertheless, in more complex situations (with scattering, real geometries, heterogeneous media), such analytical solutions are no longer available and tractable expressions such as the polynomial Eq. 3 may be useful for analysis and identification. 
In order to avoid dealing with functional forms based on averages of exponentials, it is proposed here to combine SMC with the null-collision concept. This approach, recently introduced for radiative transfer applications in $[18,19,20]$, is known to circumvent without approximation the problems generally due to the nonlinearity of the exponential term of extinction (Beer's law). In a non-scattering medium ${ }^{1}$, it consists in rewriting Eq. 1 by simply adding and subtracting a term $\gamma_{\eta} I_{\eta}$ as:

$$
\frac{d I_{\eta}}{d s}=-\hat{\beta}_{\eta} I_{\eta}+\kappa_{\eta} B_{\eta}+\gamma_{\eta} I_{\eta}
$$

where $\hat{\beta}_{\eta}=\kappa_{\eta}+\gamma_{\eta}$ is a new extinction coefficient and $\gamma_{\eta}$ is an arbitrary coefficient referred to as the null-collision coefficient. The new source term $\gamma_{\eta} I_{\eta}$ can be interpreted as a particular kind of scattering term corresponding to a pure-forward scattering event, as shown in [18]. A direct consequence of this reformulation is that the new extinction coefficient $\hat{\beta}_{\eta}$, which appears in the Beer's exponential law, can be defined arbitrarily. Accordingly, the exponentials do not depend anymore on the real radiative properties and difficulties related to nonlinearities vanish. As detailed in the next sections, Eq. 4 combined with SMC technique provide polynomial functions of the radiative quantities of interest very similar to the one given in Eq. 3 .

\section{Radiative quantities as functions of absorption coefficients}

\subsection{SMC algorithm}

The method based on the principles described previously is detailed in this section to represent the radiative intensity as a polynomial function of its absorption coefficient $\kappa_{\eta}$.

Let us consider again the case of a non-scattering uniform medium (not necessarily isothermal). The radiative intensity $I_{\eta}\left(\mathbf{x}_{0}, \mathbf{u}_{0}\right)$ at the exit of a participating medium (at location $\mathbf{x}_{0}$ and in direction $\mathbf{u}_{0}$ ) results from the intensities emitted locally all along the optical path in direction $\mathbf{u}_{0}$ (see Fig. 2) and transmitted up to $\mathbf{x}_{0}$ according to Beer's law.

A Monte Carlo algorithm for the estimation of $I_{\eta}\left(\mathbf{x}_{0}, \mathbf{u}_{0}\right)$ consists in reconstructing statistically all elementary emission events that contribute to $I_{\eta}\left(\mathbf{x}_{0}, \mathbf{u}_{0}\right)$ by the random

\footnotetext{
${ }^{1}$ Only pure absorbing/emitting medium is considered in this section for didactic purposes, but the concept of null-collision remains strictly unchanged with scattering [18].
} 


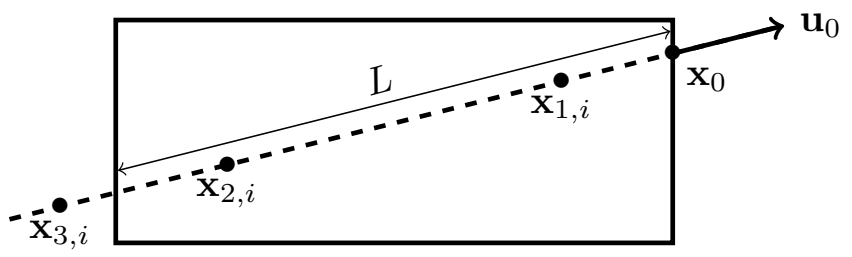

Figure 2: Example of an optical path which can be generated with the Monte Carlo algorithm. $\mathbf{x}_{1, i}, \mathbf{x}_{2, i}$ and $\mathbf{x}_{3, i}$ are the successive positions generated by the free-path sampling of the $i$-th optical path.

sampling of a large number $N$ of optical paths. For each of them, the contribution to radiative intensity noted $w_{i}$, for $i \in\{1, N\}$, is calculated. The radiative intensity is then approximated by averaging all the $N$ contributions as:

$$
I_{\eta}\left(\mathbf{x}_{0}, \mathbf{u}_{0}\right) \approx \frac{1}{N} \sum_{i=1}^{N} w_{i}
$$

In standard reverse Monte Carlo algorithms [21], starting from location $\mathbf{x}_{0}$, the generation of the $i$-th optical path consists in sampling a free-path $l_{1, i}$ according to the free-path probability density $p_{L}\left(l_{1, i}\right)=\kappa_{\eta} \exp \left(-\kappa_{\eta} l_{1, i}\right)$. An emission location $\mathbf{x}_{1, i}=$ $\mathbf{x}_{0}-l_{1, i} \mathbf{u}_{0}$ is then obtained. The value of $w_{i}$ is in this case the value of the Planck function $B_{\eta}\left(\mathbf{x}_{1, i}\right)$ at location $\mathbf{x}_{1, i}$. For more details on such algorithms, one may refer to [22] where the contributions $w_{i}$ are rigorously derived from integral formulations of the radiative transfer equation.

When null-collision approaches are applied, pure-forward scattering events which have no effect on radiative transfer modify the algorithm similarly as if scattering were considered. The following steps are required to generate randomly the $i$-th optical path. The location $\mathbf{x}_{1, i}=\mathbf{x}_{0}-l_{1, i} \mathbf{u}_{0}$ is generated randomly according to the free-path probability density which now depends on the new extinction coefficient $\hat{\beta}_{\eta}$ : $\hat{p}\left(l_{1, i}\right)=\hat{\beta}_{\eta} \exp \left(-\hat{\beta}_{\eta} l_{1, i}\right)$. At this location, two radiative sources have to be considered: true emission and pure-forward scattering events - or null-collisions - (see the positive source terms in the second member of Eq. 4). As with scattering events, null-collisions means that no emission occurs at location $\mathbf{x}_{1, i}$. This compensates the overestimated extinction coefficient $\hat{\beta}_{\eta}$ - linked to a decrease of the mean free-paths - which is higher than the real extinction coefficient given by $\kappa_{\eta}$ in non-scattering cases. From an algorithmic point of view, two situations can be considered at location $\mathbf{x}_{1, i}$ :

- In the most usual approach, a step is required to statistically determine which type of event occurs: a null-collision (with a probability given by the following 
albedo $\omega_{\eta}=\frac{\gamma_{\eta}}{\hat{\beta}_{\eta}}=\frac{\hat{\beta}_{\eta}-\kappa_{\eta}}{\hat{\beta}_{\eta}}$ ) or an emission (with probability $1-\omega_{\eta}=\frac{\kappa_{\eta}}{\hat{\beta}_{\eta}}$ ).

- In the second case, usually referred to as Energy Partitioning [19, 23], both events are deterministically considered and the contribution of each of them is weighted by the albedo $\omega_{\eta}$ for null-collision and $1-\omega_{\eta}$ for the emission.

As the objective of the present method is to retain the absorption coefficient under its symbolic form, the second approach was chosen. Indeed, in the first choice, absorption coefficient is involved in the probabilities used to determine the type of event, which leads to unnecessary methodological difficulties (which are discussed in Sec. 4.1 and in Appendix B).

Consequently, at location $\mathbf{x}_{1, i}$, emission is $\left(1-\omega_{\eta}\right) B_{\eta}\left(\mathbf{x}_{1, i}\right)$ and the contribution of a null-collision must be considered. Again, a free-path $l_{2, i}$ is sampled according to $\hat{p}_{L}\left(l_{2, i}\right)$ and a second location $\mathbf{x}_{2, i}=\mathbf{x}_{1, i}-l_{2, i} \mathbf{u}_{0}$ is determined. The contribution of emission at $\mathbf{x}_{2, i}$ becomes $\omega_{\eta}\left(1-\omega_{\eta}\right) B_{\eta}\left(\mathbf{x}_{2, i}\right)$ where $\omega_{\eta}$ indicates that a null-collision event was considered at $\mathbf{x}_{1, i}$. Now, if we assume for instance that the third location sampled according to the free-path density probability is out of the system, then the total contribution $w_{i}$ of this optical path with 2 collisions (as depicted in Fig. 2) is:

$$
w_{i}=\left(1-\omega_{\eta}\right) B_{\eta}\left(\mathbf{x}_{1, i}\right)+\omega_{\eta}\left(1-\omega_{\eta}\right) B_{\eta}\left(\mathbf{x}_{2, i}\right)
$$

For an optical path with $M_{i}$ event locations (emissions/null-collisions) inside the medium, the general formula for $w_{i}$ becomes:

$$
w_{i}=\sum_{j=1}^{M_{i}} \omega_{\eta}^{j-1}\left(1-\omega_{\eta}\right) B_{\eta}\left(\mathbf{x}_{j, i}\right)=\sum_{j=1}^{M_{i}}\left(\frac{\hat{\beta}_{\eta}-\kappa_{\eta}}{\hat{\beta}_{\eta}}\right)^{j-1}\left(\frac{\kappa_{\eta}}{\hat{\beta}_{\eta}}\right) B_{\eta}\left(\mathbf{x}_{j, i}\right)
$$

It is as a polynomial with respect to the absorption coefficient $\kappa_{\eta}$ :

$$
w_{i}=\sum_{j=1}^{M_{i}} a_{j, i} \kappa_{\eta}^{j}
$$

Following Eq. 5, the intensity at location $\mathbf{x}_{0}$ in the direction $\mathbf{u}_{0}$ can then be evaluated:

$$
I_{\eta}\left(\mathbf{x}_{0}, \mathbf{u}_{0}\right) \approx \frac{1}{N} \sum_{i=1}^{N} \sum_{j=1}^{M_{i}} a_{j, i} \kappa_{\eta}^{j}
$$

Finally, let $M_{\max }$ be the maximum number of events encountered during the $N$ random generations of optical paths and let us assume that $a_{j, i}=0$ as soon as $j>M_{i}$. Eq. 9 can be reformulated to give:

$$
I_{\eta}\left(\mathbf{x}_{0}, \mathbf{u}_{0}\right) \approx \sum_{j=1}^{M_{\max }}\left[\frac{1}{N} \sum_{i=1}^{N} a_{j, i}\right] \kappa_{\eta}^{j}=\sum_{j=1}^{M_{\max }} \overline{a_{j}} \kappa_{\eta}^{j}
$$


The strength of null-collisions concept can thus be observed directly in Eq. 10. The expression of the radiative intensity is a function of $\kappa_{\eta}$ and takes a simple polynomial form (this is due to the fact that the average of polynomial functions is also polynomial). Coefficients $\overline{a_{j}}$ are the average of the same-degree coefficients of the $N$ Monte Carlo samples: $\overline{a_{j}}=\frac{1}{N} \sum_{i=1}^{N} a_{j, i}$. Accordingly, instead of computing for each optical path (indexed $i$ ) a simple scalar sample $w_{i}$, as in standard MC approaches, $M_{\max }$ samples $a_{j, i}$ are calculated in SMC and averaged. The outputs of the SMC are in this case the coefficients of the polynomial expansion of the radiative intensity with respect to the absorption coefficient of the medium.

In non-uniform media, the principle of the method remains the same, only Eq. 7 is modified to account for the heterogeneities of the spatial absorption coefficient. This provides:

$$
w_{i}=\sum_{j=1}^{M_{i}}\left[\prod_{k=1}^{j-1} \omega\left(\mathbf{x}_{k, i}\right)\right]\left(1-\omega\left(\mathbf{x}_{j, i}\right)\right) B_{\eta}\left(\mathbf{x}_{j, i}\right)=\sum_{j=1}^{M_{i}}\left[\prod_{k=1}^{j-1} \frac{\hat{\beta}_{\eta}-\kappa_{\eta}\left(\mathbf{x}_{k, i}\right)}{\hat{\beta}_{\eta}}\right]\left(\frac{\kappa_{\eta}\left(\mathbf{x}_{j, i}\right)}{\hat{\beta}_{\eta}}\right) B_{\eta}\left(\mathbf{x}_{j, i}\right)
$$

If the radiative properties are given in a spatial grid, it is therefore possible to identify - as shown later in this paper - several absorption coefficients, or if a parametric profile for $\kappa_{\eta}$ is assumed, the approach can be used to retrieve its parameters (origin and slope if a linear profile is assumed for instance).

\subsection{Test case 1: non-scattering uniform medium}

The particular case of a non-scattering uniform and isothermal medium (of temperature $T$ ) is addressed here. Results obtained by the symbolic approach are depicted in Fig. 3 (solid line) and are compared to analytical values, represented by cross symbols. In order to treat dimensionless quantities, the ratio of the radiative intensity to the Planck blackbody intensity $I_{\eta}\left(\mathbf{x}_{0}, \mathbf{u}_{0}\right) / B_{\eta}(T)$ is considered. It is defined as a function of the absorption optical thickness $\tau_{a, \eta}=\kappa_{\eta} L$, where $L$ is the maximum path length in the medium in the direction $\mathbf{u}_{0}$ (see Fig. 2).

For this case, the arbitrary extinction coefficient $\hat{\beta}_{\eta}$ was set to obtain a global extinction thickness $\hat{\tau}_{\eta}=\hat{\beta}_{\eta} L=10$. This choice has to be made in agreement with the considered case:

- as introduced in $[18,19]$ some convergence issues can be encountered if $\hat{\beta}_{\eta}<\kappa_{\eta}$ (or alternatively if the null-collision coefficient $\gamma_{\eta}$ is negative). Therefore the 


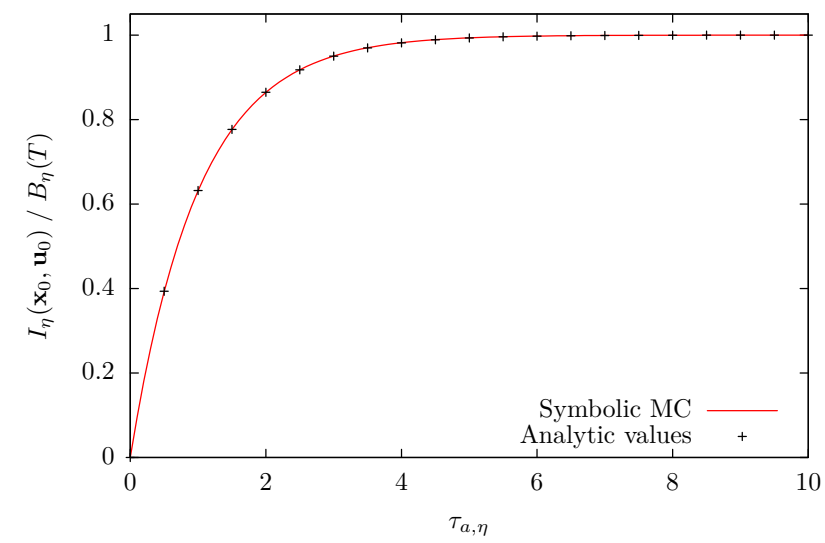

Figure 3: $I_{\eta}\left(\mathbf{x}_{0}, \mathbf{u}_{0}\right) / B_{\eta}(T)$ computed with SMC (solid line) as a function of the absorption optical thickness $\tau_{a, \eta}=\kappa_{\eta} L$ for a non-scattering uniform and isothermal medium. Location $\mathbf{x}_{0}$, direction $\mathbf{u}_{0}$ and length $L$ are described in Fig. 2, and $\hat{\tau}_{\eta}=\hat{\beta}_{\eta} L=10$. Comparison with analytical values (in cross symbols) are provided.

value of $\hat{\beta}_{\eta}$ determines the upper-bound of values of $\kappa_{\eta}$ over which the functional expression will be defined. Consequently, $\hat{\beta}_{\eta}$ must be large enough to cover the full range of absorption coefficient expected in the studied case.

- on the other hand, the higher $\hat{\beta}_{\eta}$, the higher the number of null-collisions, and the higher the degree $M_{\max }$ of the polynomial expression of $I_{\eta}$. High degree polynomials may be encountered for large values of $\hat{\tau}_{\eta}$ and can lead to complicated numerical issues (requiring for instance quadruple precision numbers, or the definition of criteria to restrict the orders of the polynomials, etc.)

Accordingly, we suggest defining the arbitrary optical thickness $\hat{\tau}_{\eta}$ as the maximum value of $\tau_{\eta}$ defined by the upper bound of the retained parameter space. Here, with $\hat{\tau}_{\eta}=10$, and $N=10^{4}$ independent optical paths, the symbolic algorithm returns the following polynomial expression:

$\frac{I_{\eta}\left(\mathbf{x}_{0}, \mathbf{u}_{0}\right)}{B_{\eta}} \approx 0.9965 \tau_{a, \eta}-0.495392 \tau_{a, \eta}^{2}+0.1638227 \tau_{a, \eta}^{3}-0.04052081 \tau_{a, \eta}^{4}+\cdots-10^{-28} \tau_{a, \eta}^{24}$

It is fully compliant with the exact analytical solution $\frac{I_{\eta}\left(\mathbf{x}_{0}, \mathbf{u}_{0}\right)}{B_{\eta}}=\left(1-\exp \left(-\kappa_{\eta} L\right)\right)$ (see Fig. 3) and is a good term-to-term approximation of its series expansion.

Finally, as for all standard Monte Carlo, the intensity can be estimated together with a confidence interval given by the standard deviation. With symbolic MC, the standard deviation is itself a function of the unknown variables: here a polynomial of degree 
$2 \times M_{\max }$ of the absorption optical thickness. Full details about its computation are provided in Appendix A. In the particular case of Fig. 3, the relative standard deviation is always lower than $0.12 \%$ for $N=10^{4}$ Monte Carlo samples.

All the previous developments can be extended without any additional methodological difficulties to account for scattering events, real geometries or for the study of others radiative quantities (such as radiative fluxes). Moreover, several unknown properties can also be identified using a single symbolic algorithm. In this case, multivariate polynomial expansions of the radiative intensity will be produced by the present technique. Examples are described in the two following test cases.

\subsection{Test cases in non-uniform scattering media}

For the two following test cases, isotropic scattering events are now considered. The objective of these cases is to show that the main interest of the considered approach is to be perfectly suited to complex configurations for which no analytical solution is available. The algorithm is slightly modified to account for these new collisions: for each Monte Carlo sample, a multiple-scattering path is first generated according to a given scattering coefficient, then the radiative computation leading to $w_{i}$ (which involves emissions and null-collisions) is applied along the curvilinear path exactly in the same way as before. The considered medium is now non-uniform and made of two infinite and parallel slabs $\mathcal{M}_{1}$ and $\mathcal{M}_{2}$ as depicted in Fig. 4. For each layer the scattering optical thickness is set to $\tau_{s, \eta}=\sigma_{\eta, 1} L_{1}=\sigma_{\eta, 2} L_{2}=0.5$.

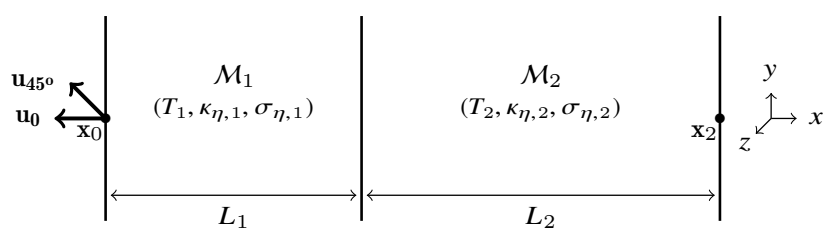

Figure 4: Medium composed of two homogeneous columns $\mathcal{M}_{1}$ and $\mathcal{M}_{2}$ of respective widths $L_{1}$ and $L_{2}$, temperatures $T_{1}$ and $T_{2}$, absorption coefficients $\kappa_{\eta, 1}$ and $\kappa_{\eta, 2}$, and scattering coefficients $\sigma_{\eta, 1}$ and $\sigma_{\eta, 2}$.

\subsubsection{Test case 2: radiative intensity as a function of two absorption coefficients}

In this case, the temperatures of the slabs were set in such a way that the Planck blackbody functions in $\mathcal{M}_{2}$ is 10 times higher than in $\mathcal{M}_{1}$. Both absorption coefficients 
$\kappa_{\eta, 1}$ and $\kappa_{\eta, 2}$ are supposed to be unknown. Under these considerations, only two values of absorption coefficients are considered, and the polynomial expression of the sample $w_{i}$ - directly derived from Eq. 11 - can be expressed as a bivariate polynomial:

$$
w_{i}=\sum_{p=0}^{M_{1, i}} \sum_{q=0}^{M_{2, i}} b_{p, q, i} \kappa_{\eta, 1}^{p} K_{\eta, 2}^{q}
$$

where $M_{1, i}$ and $M_{2, i}$ are the number of collisions that occur, for the $i$-th optical path, in slabs $\mathcal{M}_{1}$ and $\mathcal{M}_{2}$ respectively. Coefficients $b_{p, q, i}$ are numerical values computed during the simulation. The ratio $I_{\eta}\left(\mathbf{x}_{0}, \mathbf{u}_{0}\right) / B_{\eta}\left(T_{2}\right)$ calculated as the average of the $N$ functional samples $w_{i}$ is also a bivariate polynomial. It is shown in Fig. 5. In this plot,

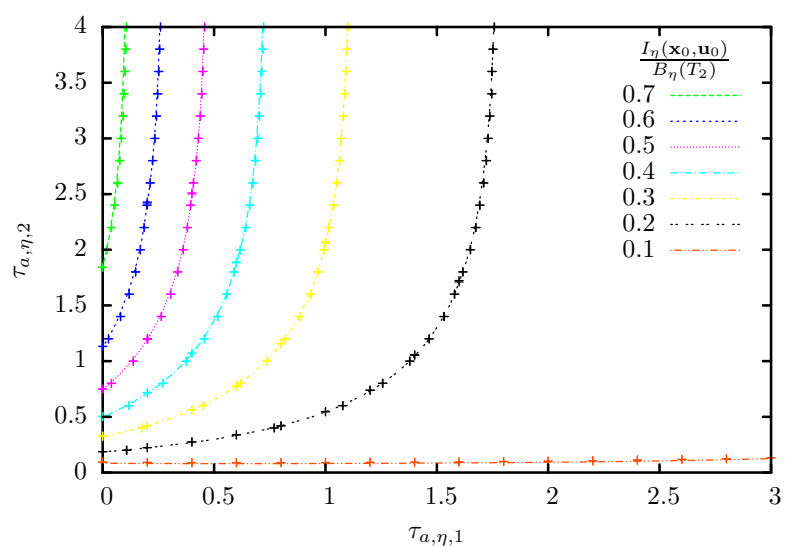

Figure 5: $I_{\eta}\left(\mathbf{x}_{0}, \mathbf{u}_{0}\right) / B_{\eta}\left(T_{2}\right)$ as a function of absorption optical thicknesses $\tau_{a, \eta, 1}=\kappa_{\eta, 1} L_{1}$ and $\tau_{a, \eta, 2}=\kappa_{\eta, 2} L_{2}$ for a scattering medium composed of two parallel and infinite slabs (see Fig. 4). Results obtained with SMC (solid lines) and standard MC results (cross symbols) are presented. $\hat{\tau}_{\eta}$ is set to 10 .

each isoline represents the infinity of couples $\left(\tau_{a, \eta, 1}, \tau_{a, \eta, 2}\right)$ which leads to the same ratio $I_{\eta}\left(\mathbf{x}_{0}, \mathbf{u}_{0}\right) / B_{\eta}\left(T_{2}\right)$. Consequently, every couples $\left(\tau_{a, \eta, 1}, \tau_{a, \eta, 2}\right)$ on these isolines do not have necessarily a physical meaning. They only represent one possible solution of the RTE for a given value of $I_{\eta}\left(\mathbf{x}_{0}, \mathbf{u}_{0}\right) / B_{\eta}\left(T_{2}\right)$.

This figure can be used in an identification context. If no prior information is available, several experimental values $I_{\eta}^{\text {meas }}$ are required to identify a single couple of absorption coefficients. For instance, if we have at our disposal experimental values for several locations and directions, it is possible to plot multiple curves in the same figure, as shown in Fig. 6, and to use the coordinates at which the curves intercept to find the solution.

Indeed, the three curves corresponding to the isolines of the experimental values 


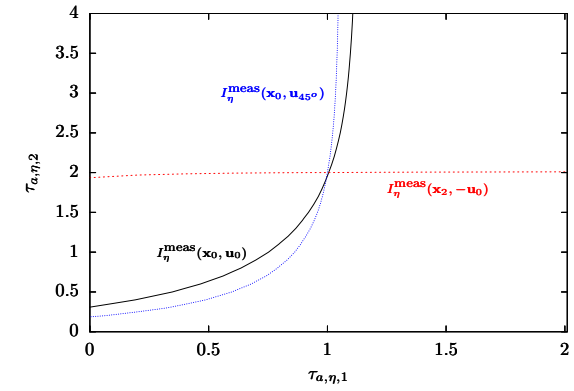

(a) without experimental uncertainty

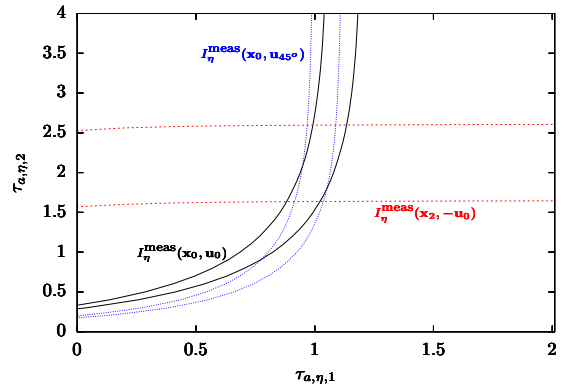

(b) with $5 \%$ of experimental uncertainties

Figure 6: $I_{\eta}\left(\mathbf{x}_{0}, \mathbf{u}_{0}\right) / B_{\eta}\left(T_{2}\right), I_{\eta}\left(\mathbf{x}_{0}, \mathbf{u}_{45^{\circ}}\right) / B_{\eta}\left(T_{2}\right)$ and $I_{\eta}\left(\mathbf{x}_{2},-\mathbf{u}_{0}\right) / B_{\eta}\left(T_{2}\right)$ as function of absorption optical thicknesses $\tau_{a, \eta, 1}=\kappa_{\eta, 1} L_{1}$ and $\tau_{a, \eta, 2}=\kappa_{\eta, 2} L_{2}$. Only isolines corresponding to the pseudo-measured intensities are represented for each function. The locations $\mathbf{x}_{0}$ and $\mathbf{x}_{2}$, and the directions $\mathbf{u}_{0}$ and $\mathbf{u}_{45^{\circ}}$ are described in Fig. 4. $\hat{\tau}_{\eta}$ is set to 10 .

for three intensities intersect in Fig. 6a at a point which corresponds to the unknown couple of properties $\tau_{a, \eta, 1}, \tau_{a, \eta, 2}\left(\tau_{a, \eta, 1}=1, \tau_{a, \eta, 2}=2\right.$ in this case $)$. In practice these curves overlap over a small region that encompasses the intersection point because of the stochastic nature of Monte Carlo algorithms. However, this small region can be readily determined by the respective confidence intervals of each curve (estimated through the computation of standard deviations, see Appendix A). If necessary, the accuracy of the retrieved values of $\tau_{a, \eta, 1}$ and $\tau_{a, \eta, 2}$ can be improved by increasing the number $N$ of independent samples.

In figure $6 \mathrm{~b}$, it is assumed that the pseudo-measurements are subject to $5 \%$ experimental uncertainties. Therefore, for each pseudo-measured radiative intensity, two isolines corresponding to $0.95 I_{\eta} / B_{\eta}\left(T_{2}\right)$ et $1.05 I_{\eta} / B_{\eta}\left(T_{2}\right)$ are displayed. In this case, the SMC polynomial gives the region covering the couples of properties that are potential solutions of the inverse problem. In this particular case, a second measurement at location $\mathbf{x}_{2}$ in the direction $-\mathbf{u}_{0}$ will give more additional information than at $\mathbf{x}_{0}$ in a $45^{\circ}$ direction. Indeed the region of potential solutions is much smaller when $I_{\eta}\left(\mathbf{x}_{0}, \mathbf{u}_{0}\right) / B_{\eta}\left(T_{2}\right)$ is considered together with $I_{\eta}\left(\mathbf{x}_{2},-\mathbf{u}_{0}\right) / B_{\eta}\left(T_{2}\right)$. The experimental values of $I_{\eta}\left(\mathbf{x}_{0}, \mathbf{u}_{45^{\circ}}\right)$ are very close to $I_{\eta}\left(\mathbf{x}_{0}, \mathbf{u}_{0}\right)$, and consequently do not bring new relevant information for the identification of the radiative properties.

Fig. 6 shows that the functional expressions of $I_{\eta}$ can be of great interest for experimental design or to gather information for inversion purposes. 
3.3.2. Test case 3: radiative intensity as a function of absorption coefficient and temperature

It is also possible to express the radiative intensity as a function of absorption coefficients and temperatures. We consider now - keeping the same configuration as in Fig. 4 - that the absorption optical thickness $\tau_{a, \eta, 1}=\kappa_{\eta, 1} L_{1}$ of the first slab is known as well as its temperature $T_{1}$. The absorption coefficient $\kappa_{\eta, 2}$ and the temperature $T_{2}$ of the medium $\mathcal{M}_{2}$ are unknown. The sample $w_{i}$ given by Eq. 11 can be expressed under the polynomial form:

$$
w_{i}=c_{i}+\sum_{q=1}^{M_{2, i}}\left[d_{q, i} \kappa_{\eta, 2}^{q} B_{\eta}\left(T_{2}\right)\right]
$$

where $M_{2, i}$ is the number of collisions that occurred, for the $i$-th sampling, in slab $\mathcal{M}_{2}$, and $c_{i}$ and $d_{q, i}$ are numerical coefficients derived from Eq. 11.

Fig. 7 represents isolines for the infinity of couples $\left(\tau_{a, \eta, 2}, T_{2}\right)$ that provide - according to the radiative transfer equation - a given value of $I_{\eta}\left(\mathbf{x}_{0}, \mathbf{u}_{0}\right) / B_{\eta}\left(T_{1}\right)$. Again,

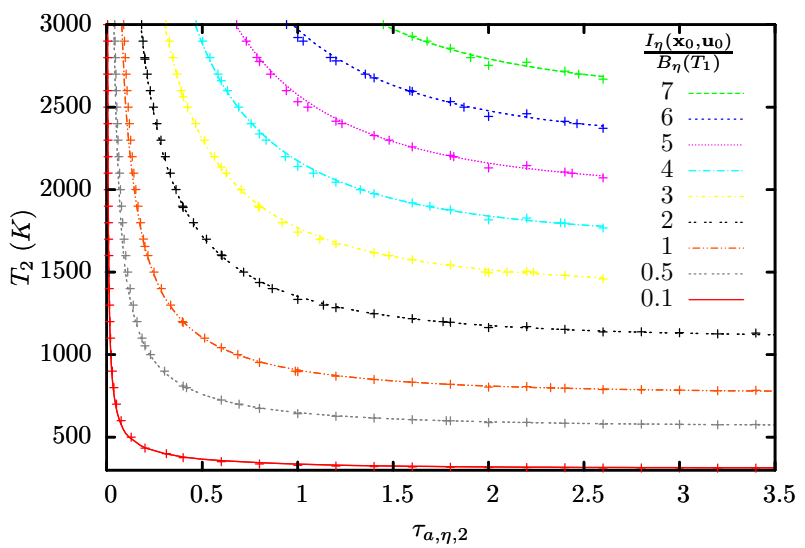

Figure 7: $I_{\eta}\left(\mathbf{x}_{0}, \mathbf{u}_{0}\right) / B_{\eta}\left(T_{1}\right)$ as function of absorption optical thickness $\tau_{a, \eta, 2}=\kappa_{\eta, 2} L_{1}$ and temperature $T_{2}$ for a scattering medium composed of two parallel and infinite slabs (see Fig. 4). Results obtained with SMC (solid lines) can be assessed against standard MC results (cross symbols). $\hat{\tau}_{\eta, 2}$ is set to 6 .

all points on these isolines do not have necessarily a true physical meaning. If a prior knowledge about the temperature-dependence of the absorption coefficient is available with respect to the case study, both the temperature and the absorption coefficient can be retrieved from one or several experimental measurements (it depends on whether the solution is unique or not). For instance, in a gaseous medium, line-by-line calculations may provide the evolution of the absorption coefficient with temperature. Similarly to the curves presented in Fig. 6, the intersection between the SMC and the line-by-line 
curves may provide the solution $\left(\tau_{a, \eta, 2}, T_{2}\right)$. In the same logic, from a single symbolic simulation, such results can be also used to indirectly identify some intrinsic properties of the considered participating medium (such as mole fractions for gaseous mixtures). The proposed SMC algorithm is therefore very promising for problems related to the identification of temperature and molecular species concentrations in gaseous systems.

\section{Radiative quantities as functions of scattering coefficients}

In the previous section, the scattering coefficient $\sigma_{\eta}$ was supposed to be known. It was therefore possible to divide each sampling of the MC algorithm in two steps: a multiple-scattering path was first generated according to $\sigma_{\eta}$, and then the radiative transfer computation was performed along this path. However, if the scattering coefficient is unknown, or aimed to be kept under a symbolic formalism, the first stage of curvilinear path generation is no longer feasible. The three sources of radiation, emission, scattering and null-collision, must be simultaneously taken into account during the radiative transfer computation. Handling this kind of situation is the object of the next paragraphs, devoted to the extension of SMC technique to radiative intensities as functions of scattering coefficients or as functions of both scattering and absorption coefficients.

\subsection{SMC algorithm}

Let us consider a uniform emitting, absorbing and scattering medium of finite dimensions. The intensity $I_{\eta}\left(\mathbf{x}_{0}, \mathbf{u}_{0}\right)$ at location $\mathbf{x}_{0}$, in the direction $\mathbf{u}_{0}$ results from intensities emitted along multiple-scattering paths. Here again, the Monte Carlo algorithm consists in reconstructing statistically all these contributions along the optical path.

Standard reverse Monte Carlo algorithms (without null-collisions) perform a large number $N$ of independent realizations (indexed $i$ ): each of them is composed of successive samplings of free-paths with respect to the probability density $p_{L}(l)=\beta_{\eta} \exp \left(-\beta_{\eta} l\right)$, where $\beta_{\eta}=\kappa_{\eta}+\sigma_{\eta}$ is the extinction coefficient [21]. Along the $i$-th optical path, the type of event occurring at each source location $\mathbf{x}_{j, i}$ may be determined in a stochastic way. The probabilities of emission $P_{e}=\kappa_{\eta} / \beta_{\eta}$ and scattering $P_{s}=1-P_{e}=\sigma_{\eta} / \beta_{\eta}$ are then used to determine if emission or scattering occurs. If a scattering event is randomly generated, a new direction is sampled according to the phase function, and a new 
source location $\mathbf{x}_{j+1, i}$ is sampled according to $p_{L}(l)$. If it is an emission, the contribution to $I_{\eta}\left(\mathbf{x}_{0}, \mathbf{u}_{0}\right)$ is defined as the local blackbody Planck function: $w_{i}=B_{\eta}\left(\mathbf{x}_{j, i}\right)$.

However, as explained in Sec. 2 and 3, such standard approaches can hardly be used within a symbolic context because the exponential extinction depends on both $\sigma_{\eta}$ and $\kappa_{\eta}$ and leads to intractable functional expressions for the radiative quantities. Again, null-collisions can be introduced to overcome this difficulty. The algorithm is then slightly modified to include null-collisions, as detailed in [18]: the probability density of free paths is now $\hat{p}_{L}(l)=\hat{\beta}_{\eta} \exp \left(-\hat{\beta}_{\eta} l\right)$ where the new extinction coefficient is $\hat{\beta}_{\eta}=\kappa_{\eta}+\sigma_{\eta}+\gamma_{\eta}$. This quantity includes henceforth the null-collision coefficient $\gamma_{\eta}$. Once again, two choices are possible from an algorithmic point of view:

- In the first choice, three types of events can be stochastically determined at each source location $\mathbf{x}_{j, i}$ : absorption or scattering - as in standard algorithm or null-collisions with respective probabilities $P_{e}=\kappa_{\eta} / \hat{\beta}_{\eta}, P_{s}=\sigma_{\eta} / \hat{\beta}_{\eta}$ and $P_{n}=1-P_{e}-P_{s}=\frac{\beta_{\eta}-\kappa_{\eta}-\sigma_{\eta}}{\hat{\beta}_{\eta}}$. If a null-collision occurs, the considered direction remains unchanged and a new source location $\mathbf{x}_{j+1, i}$ is sampled according to $\hat{p}_{L}(l)$. The optical path is then generated until an emission occurs, or until it exits the medium.

- In the second choice, an energy partitioning approach can be alternatively employed as proposed in [19]. It means that, for each source location, a weighted emission contribution $\left(\kappa_{\eta} / \hat{\beta}_{\eta}\right) B_{\eta}\left(\mathbf{x}_{j, i}\right)$ is taken into account for the estimation of $I_{\eta}$. Then, the type of scattering: real scattering or null-collision, is stochastically identified with respective probabilities $\tilde{P}_{s}=\frac{\sigma_{\eta}}{\hat{\beta}_{\eta}-\kappa_{\eta}}=\frac{\sigma_{\eta}}{\sigma_{\eta}+\gamma_{\eta}}$ and $\tilde{P}_{n}=1-\tilde{P}_{s}=$ $\frac{\beta_{\eta}-\kappa_{\eta}-\sigma_{\eta}}{\hat{\beta}_{\eta}-\kappa_{\eta}}=\frac{\gamma_{\eta}}{\sigma_{\eta}+\gamma_{\eta}}$. The optical path generation ends at the exit of the system.

In the symbolic approach, where the scattering coefficient is unknown, the energy partitioning approach is a better option for similar reasons as in Sec. 3. Probabilities $P_{e}, P_{s}$ and $P_{n}=1-P_{s}-P_{a}$ are unknown at $\mathbf{x}_{j, i}$, which means that two probabilities ( $\tilde{P}_{e}$ and $\tilde{P}_{s} ; \tilde{P}_{n}=1-\tilde{P}_{e}-\tilde{P}_{s}$ is then deduced) must be chosen arbitrarily if the first method is used. The arbitrary probabilities are introduced using the same approach as in importance sampling techniques [1] frequently used to optimize, without bias, Monte Carlo algorithms. In the second method (energy partitioning), only one arbitrary probability $\left(\tilde{P}_{s} ; \tilde{P}_{n}=1-\tilde{P}_{s}\right.$ is then deduced) is required to determine the type of scattering event. The influence of the choice of $\tilde{P}_{s}$ is discussed in Appendix B. In the 
following results, $\tilde{P}_{s}$ and $\tilde{P}_{n}$ were fixed to 0.5 , i.e., real scattering and null-collisions are assumed equiprobable.

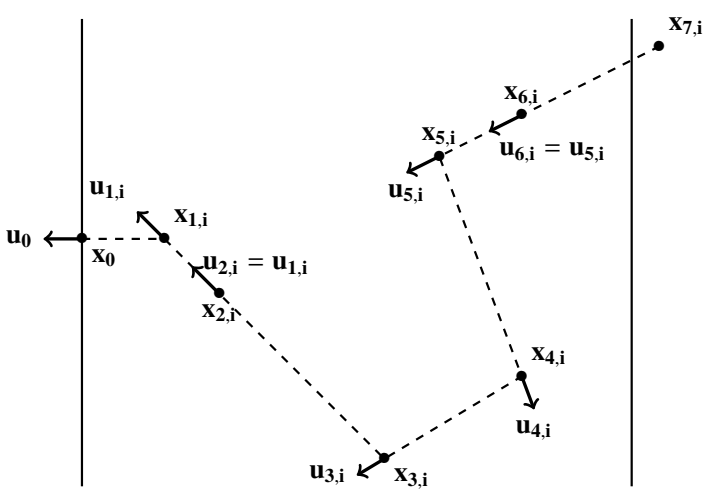

Figure 8: Example of optical path in a medium where scattering and null-collision events are considered. Starting from $\mathbf{x}_{0}$, a multiple-scattering path is generated through the sampling of free-path according to $\hat{p}_{L}\left(l_{j, i}\right)$. These paths define source locations where a type of scattering event must be defined: a null collision (as in $\mathbf{x}_{2, i}$ and $\mathbf{x}_{6, i}$, where no change of direction is applied) or a real scattering event (as in $\mathbf{x}_{1, i}, \mathbf{x}_{3, i}, \mathbf{x}_{4, i}$ and $\mathbf{x}_{5, i}$, where the optical path direction is modified according to the phase function). $\mathbf{x}_{7, i}$ is outside of the considered system, and the $i$-th optical path random generation is therefore stopped.

In order to express the radiative intensity $I_{\eta}\left(\mathbf{x}_{0}, \mathbf{u}_{0}\right)$ as a function of $\sigma_{\eta}$ and $\kappa_{\eta}$, the SMC algorithm consists in generating stochastically a large number $N$ of optical paths from location $\mathbf{x}_{0}$ in the direction $-\mathbf{u}_{0}$. Each location $\mathbf{x}_{j, i}$ along the $i$-th optical path at which a collision occurs is sampled according to the probability density of free-paths $\hat{p}(l)$ (independent on $\kappa_{\eta}$ and $\sigma_{\eta}$ ). The fraction of emission at $\mathbf{x}_{j, i}$ that is added to the $i$ th optical path contribution is $\frac{k_{\eta}}{\hat{\beta}_{\eta}} B_{\eta}\left(\mathbf{x}_{j, i}\right)$. Then the type of scattering event is sampled according to $\tilde{P}_{s}$ and $\tilde{P}_{n}$. If a real scattering event is sampled, its contribution is $\frac{\sigma_{\eta}}{\hat{\beta}_{\eta} \tilde{P}_{s}}$. If a null-collision occurs, it is $\frac{\hat{\beta}_{\eta}-\kappa_{\eta}-\sigma_{\eta}}{\hat{\beta}_{\eta} \tilde{P}_{n}}$. The optical path is generated with its multiple scattering events until it exits the medium (see figure 8).

The total contribution of an optical path $i$ with $M_{n c, i}$ null-collisions and $M_{s, i}$ scattering events is then:

$$
w_{i}=\sum_{j=1}^{M_{n c, i}+M_{s, i}} \frac{\kappa_{\eta}}{\hat{\beta}_{\eta}} B_{\eta}\left(\mathbf{x}_{j, i}\right)\left(\frac{\hat{\beta}_{\eta}-\kappa_{\eta}-\sigma_{\eta}}{\hat{\beta}_{\eta} \tilde{P}_{n}}\right)^{N_{n c, j, i}}\left(\frac{\sigma_{\eta}}{\hat{\beta}_{\eta} \tilde{P}_{s}}\right)^{N_{s, j, i}}
$$

where $N_{n c, j, i}$ and $N_{s, j, i}$ are the number of null-collisions and true scattering events which have respectively occurred before the $j$ th collision along the $i$ th optical path $\left(N_{n c, j, i}+\right.$ $\left.N_{s, j i}=j-1\right)$. 
In non-uniform media, the principle remains the same. Only Eq. 15 is modified to account for the absorption and scattering coefficients heterogeneities (similarly to Eq. 11 in Sec 3):

$w_{i}=\sum_{j=1}^{M_{n c, i}+M_{s, i}} \frac{\kappa_{\eta}\left(\mathbf{x}_{j, i}\right)}{\hat{\beta}_{\eta}} B_{\eta}\left(\mathbf{x}_{j, i}\right)\left[\prod_{k=1}^{j-1}\left\{\left(1-\mathcal{S}\left(\mathbf{x}_{k, i}\right)\right) \frac{\hat{\beta}_{\eta}-\kappa_{\eta}\left(\mathbf{x}_{k, i}\right)-\sigma_{\eta}\left(\mathbf{x}_{k, i}\right)}{\hat{\beta}_{\eta} \tilde{P}_{n}}+\mathcal{S}\left(\mathbf{x}_{k, i}\right) \frac{\sigma_{\eta}\left(\mathbf{x}_{k, i}\right)}{\hat{\beta}_{\eta} \tilde{P}_{s}}\right\}\right]$

where $\mathcal{S}\left(\mathbf{x}_{k, i}\right)=1$ if a real scattering event occurs at location $\mathbf{x}_{k, i}$, and $\mathcal{S}\left(\mathbf{x}_{k, i}\right)=0$ if it is a null-collision. Starting from Eq. 16, several values of scattering and/or absorption coefficients may be identified with the SMC.

Eqs. 15 and 16 can be rigorously demonstrated from integral formulations of the RTE. More details about such approaches where Monte Carlo algorithms are derived from integrals can be found in $[18,22]$.

4.2. Test case 4: Radiative intensity as a function of the scattering coefficient in homogeneous media

If the absorption coefficient $\kappa_{\eta}$ is known, Eq.15 is a polynomial function with respect to $\sigma_{\eta}$ :

$$
w_{i}=\sum_{j=0}^{M_{n c, i}+M_{s, i}-1} a_{j, i} \sigma_{\eta}^{j}
$$

and the radiative intensity is:

$$
I_{\eta}\left(\mathbf{x}_{0}, \mathbf{u}_{0}\right) \approx \sum_{j=0}^{M_{\max }-1} \overline{a_{j}} \sigma_{\eta}^{j}
$$

where $\overline{a_{j}}=\frac{1}{N} \sum_{i=1}^{N} a_{j, i}$, and $M_{\max }$ is the maximum number of collisions (including scattering and null-collisions) encountered during the $N$ random generations of optical paths, and assuming that $a_{j, i}=0$ if $j>\left(M_{n c, i}+M_{s, i}-1\right)$.

Figure 9 displays the radiative intensity at the exit of an absorbing and scattering slab versus the scattering optical thickness $\tau_{s, \eta}=\sigma_{\eta} L$. Comparisons with standard computation are also provided. Each cross symbol corresponds to one standard MC simulation, while only one symbolic calculation is required to obtain the SMC curve.

The relative standard deviations are very weak between $\tau_{s, \eta}=1$ and $\tau_{s, \eta}=3.5$ (smaller than $0.11 \%$ ). Beyond these values, variances slightly increase (until $0.27 \%$ ) because of the choice of $\tilde{P}_{s}=0.5$ as discussed in Appendix B. Furthermore, as already mentioned in Sec. 2, the polynomial function provided by the SMC is a useful tool for designing experimental devices and defining an identification strategy. For instance, in 


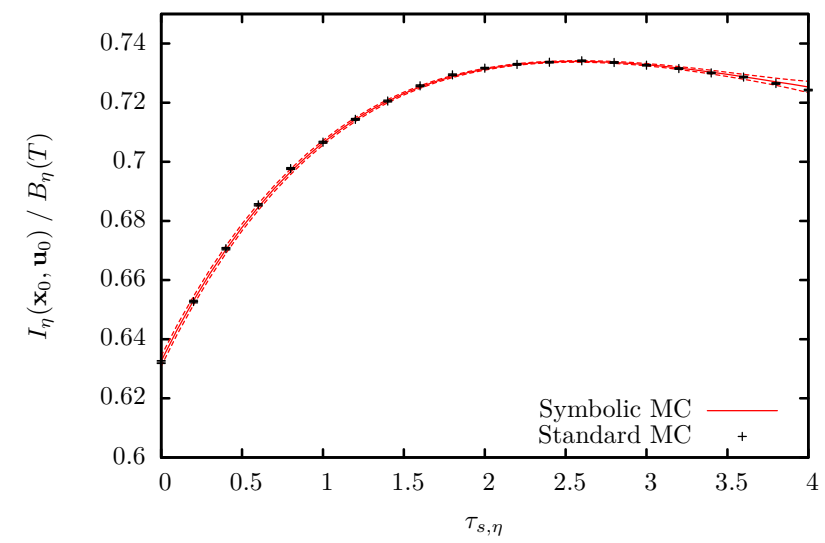

Figure 9: $I_{\eta}\left(\mathbf{x}_{0}, \mathbf{u}_{0}\right) / B_{\eta}(T)$ as function of the scattering optical thickness $\tau_{s, \eta}=\sigma_{\eta} L$ in a uniform and isothermal medium. The SMC curve (solid line) is given with its confidence interval (dotted lines) for $N=10^{6}$ samples of optical paths. Standard MC results are also displayed (cross symbols). A HenyeyGreenstein phase function has been used with an asymmetry factor $g=0.3$. The absorption optical thickness $\tau_{a, \eta}=\kappa_{\eta} L$ is fixed to 1 , and the total optical thickness (including null-collision) $\hat{\tau}_{\eta}=\hat{\beta}_{\eta} L$ is set to 20 . $\tilde{P}_{s}=\tilde{P}_{n}=0.5$.

the case of figure 9 , if the measured intensity $I_{\eta}^{\text {meas }}\left(\mathbf{x}_{0}, \mathbf{u}_{0}\right) / B_{\eta}(T)$ is lower than 0.7 , the scattering coefficient can be readily identified using the polynomial, and with strong confidence since the SMC curve indicates that the solution is unique for this intensity.

\subsection{Test case 5: radiative intensity as a function of the absorption and scattering} coefficients in homogeneous media

When more than one parameter has to be kept under a symbolic form, the SMC algorithm proposed in Sec. 4.2 leads to multivariate polynomial expansions of the radiative quantities. If both $\kappa_{\eta}$ and $\sigma_{\eta}$ are unknown, a multivariate polynomial expression can be derived from Eq.15 :

$$
w_{i}=\sum_{j=0}^{M_{n c, i}+M_{s, i}-1} \sum_{k=0}^{M_{n c, i}} b_{j, k, i} \sigma_{\eta}^{j} \kappa_{\eta}^{k}
$$

where $b_{j, k, i}$ is computed numerically with the SMC. The radiative intensity is then obtained, following the same steps as described in section 3 :

$$
I_{\eta}\left(\mathbf{x}_{0}, \mathbf{u}_{0}\right) \approx \sum_{j=0}^{M_{n c, \max }+M_{s, \max }-1} \sum_{k=0}^{M_{n c, \max }} \overline{b_{j, k}} \sigma_{\eta}^{j} \kappa_{\eta}^{k}
$$

where $\overline{b_{j, k}}=\frac{1}{N} \sum_{i=1}^{N} b_{j, k, i}, M_{n c, \max }$ and $M_{s, \max }$ are the maximum number of null-collision and scattering events encountered during the $N$ random generations of optical paths, 
and assuming that $b_{j, k, i}=0$ when $j>\left(M_{n c, i}+M_{s, i}-1\right)$ or $k>M_{n c, i}$.

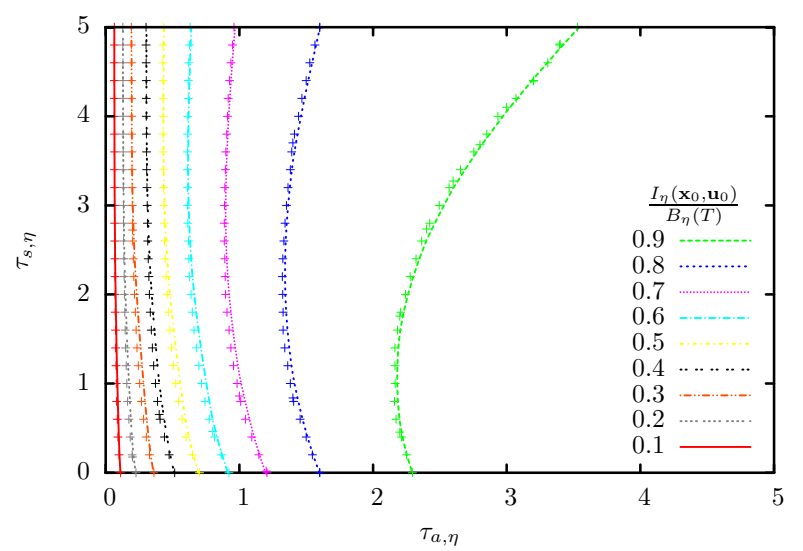

Figure 10: $I_{\eta}\left(\mathbf{x}_{0}, \mathbf{u}_{0}\right) / B_{\eta}(T)$ as a function of the absorption and scattering optical thickness $\tau_{a, \eta}=\kappa_{\eta} L$ and $\tau_{s, \eta}=\sigma_{\eta} L$ in a uniform and isothermal medium. $\hat{\tau}_{\eta}$ is set to $\hat{\beta}_{\eta} L=10$ and $\tilde{P}_{s}=\tilde{P}_{n}=0.5$. SMC curves (for $N=10^{6}$ ) are depicted in solid line. Standard MC results are displayed in cross symbols.

An example of results is provided in figure 10 where a uniform absorbing and scattering slab is considered. The Henyey-Greenstein phase function with an asymmetry factor $g=0.3$ was used. Fig. 10 represents isolines for the infinity of couples $\left(\tau_{a, \eta}, \tau_{s, \eta}\right)$ that lead - according to the RTE - to a given value of $I_{\eta}\left(\mathbf{x}_{0}, \mathbf{u}_{0}\right) / B_{\eta}(T)$. Comparisons with standard MC show that SMC accurately predicts the isolines. The average of the absolute difference between SMC and MC computations of $I_{\eta}\left(\mathbf{x}_{0}, \mathbf{u}_{0}\right) / B_{\eta}(T)$ is $1.9 \times 10^{-3}$. The maximum absolute difference is 0.019 .

The isolines depicted in Fig. 10 show the difficulty to obtain relevant information about the scattering coefficient if the measured intensity is lower than 0.8 . On the other hand, the absorption coefficient can be identified easily, especially if $I_{\eta}^{\text {meas }}\left(\mathbf{x}_{0}, \mathbf{u}_{0}\right) / B_{\eta}(T)$ is lower than 0.5 , i.e., if the medium is optically thin in terms of absorption. Obviously, only one measurement of the radiative intensity is not sufficient in this case to identify two parameters. A priori information or measurements of other radiative quantities (in other directions or at distinct locations, or for other wavenumbers) are therefore required to ensure the feasibility of the identification process. 


\section{Application to the identification of absorption and scattering coefficients in multi-layered heterogeneous media}

Complex heterogeneous media such as porous materials (ceramic or metallic foams, or fibrous structures) are generally made of a semi-transparent or opaque structures with pores filled with transparent or semi-transparent gazes. In those complex twophase media, identifying the radiative properties of the solid structure and/or the gas in the pore remains a challenging task [11]. Eventually, models based on homogenized properties have been proposed $[24,25]$ to allow the characterization of this kind of media treated in the limit of geometric optics.

The aim of the present section is to show that SMC can be used in heterogeneous media. Here, an academic problem, representative of the difficulty imposed by the heterogeneous nature of porous media, is investigated. The objective is to express the radiative intensity as a function of the absorption and scattering coefficients of a semitransparent medium (for instance the solid part of the porous structure), assuming that the radiative properties of the gases in the pores are known.

A multi-layered slab (with 20 layers of random widths) made of two absorbing and scattering media is considered (see figure 11). A Henyey-Greenstein phase function with an asymmetry factor $g=0.3$ is retained.

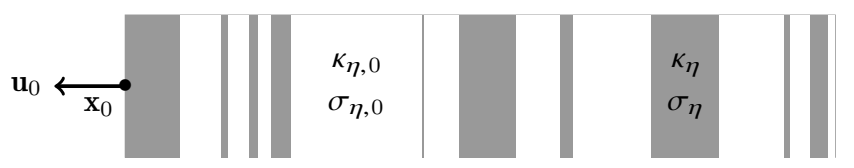

Figure 11: Scheme of the multi-layered slab with random layer's widths. $\kappa_{\eta, 0}$ and $\sigma_{\eta, 0}$ are supposed to be known, and the SMC algorithm is used to express the radiative intensity as a function of $\kappa_{\eta}$ and $\sigma_{\eta}$. The sizes (in mm) of the layers are (starting from the left boundary) : $L_{1}=0.768, L_{2}=0.584, L_{3}=0.0935$, $L_{4}=0.292, L_{5}=0.139, L_{6}=0.178, L_{7}=0.273, L_{8}=1,86, L_{9}=0.0244, L_{10}=0.491, L_{11}=0.805$, $L_{12}=0.622, L_{13}=0.175, L_{14}=1.11, L_{15}=0.954, L_{16}=0.918, L_{17}=0.0882, L_{18}=0.270, L_{19}=0.264$, $L_{20}=0.0921$.

The heterogeneous nature of the porous medium is taken into account by the multilayered configuration, since a multi-scattering optical path will cross the two different media in the same way as if a real 3D heterogeneous medium was considered. Refraction and reflection at the interface between the two media are not taken into account here, but can also be added in the generation of optical paths without additional methodological difficulties. 
Figure 12 displays the isolines of $I_{\eta}\left(\mathbf{x}_{0}, \mathbf{u}_{0}\right) / B_{\eta}(T)$ versus the absorption and scattering coefficients $\kappa_{\eta}$ and $\sigma_{\eta}$ for the considered multi-layered slab of $1 \mathrm{~cm}$ width. The degrees of the SMC bivariate polynomials is $92 \times 52$, i.e., $I_{\eta}\left(\mathbf{x}_{0}, \mathbf{u}_{0}\right) \approx \sum_{j=0}^{92} \sum_{k=0}^{52} \overline{b_{j, k}} \sigma_{\eta}^{j} \kappa_{\eta}^{k}$.

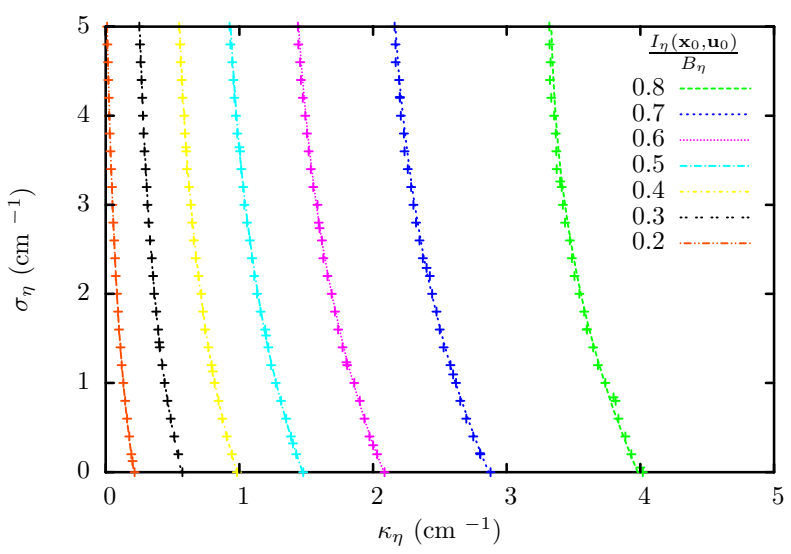

Figure 12: $I_{\eta}\left(\mathbf{x}_{0}, \mathbf{u}_{0}\right) / B_{\eta}(T)$ as a function of the absorption and scattering coefficients $\kappa_{\eta}$ and $\sigma_{\eta}$. SMC (solid lines) and standard Monte Carlo (cross symbols) simulations are displayed. The extinction optical thickness is set to $\hat{\beta}_{\eta} L=10$ and $\tilde{P}_{s}=\tilde{P}_{n}=0.5$. The known absorption and scattering coefficients are set as $\kappa_{\eta, 0}=\sigma_{\eta, 0}=0.2 \mathrm{~cm}^{-1}$.

Comparison with standard MC shows that the SMC polynomial provides an accurate fit of the isolines. The average of the absolute difference between SMC and MC computations of $I_{\eta}\left(\mathbf{x}_{0}, \mathbf{u}_{0}\right) / B_{\eta}(T)$ is $1.7 \times 10^{-3}$. The maximum absolute difference is 0.021. The ratio of the computational times between one SMC and one standard MC simulations varies between $t_{S M C} / t_{M C}=4.3$ (when $t_{M C}$ is considered for the maximum values of $\sigma_{\eta}$ and $\kappa_{\eta}$ ) and 88.4 (for the minimum values of $\sigma_{\eta}$ and $\kappa_{\eta}$ ). In average, this ratio is about 7.8 . For a single simulation, $t_{S M C}$ is higher than $t_{M C}$ for two reasons : 1/ the introduction of null-collisions increases the computational cost [18] and 2/ numerous polynomial coefficients are estimated with SMC (including the coefficients needed for the standard deviation), while only two scalar values (one for the intensity plus one for the standard deviation) are estimated with standard MC. However, only one single SMC simulation is needed to solve the RTE over the whole parameter space, here $[0,5] \times[0,5]$. Using standard MC, $50 \times 50$ simulations were carried out to cover the same parameter space, resulting in a computational cost 322 times higher than SMC.

Similarly to the results presented in section 4.3 , measuring $I_{\eta}\left(\mathbf{x}_{0}, \mathbf{u}_{0}\right)$ does not allow obtaining significant information on scattering, but if the medium is optically thin in 
absorption, the absorption coefficient can be identified without difficulty.

A remarkable feature of the SMC polynomials is to enable a straightforward development of sensitivity analysis. Indeed, the polynomials can be easily derived to calculate any order sensitivity or cross-sensitivity, without additional computational cost, which may be relevant for inversion, or to get physical insight about the influence of the considered parameters.

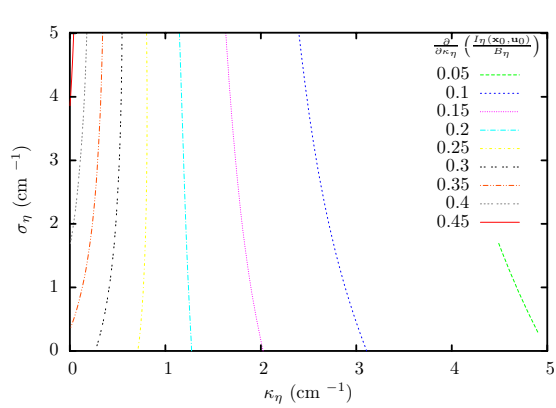

(a) Sensitivity to $\kappa_{\eta}$

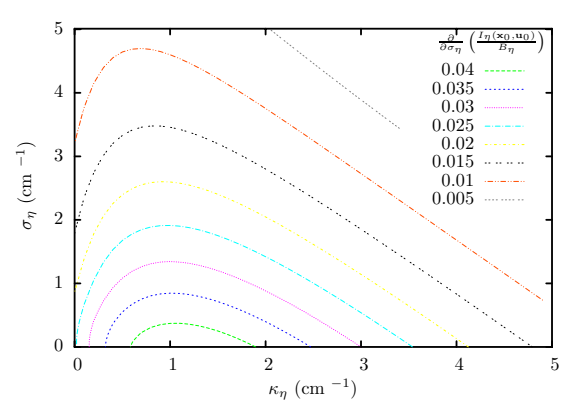

(b) Sensitivity to $\sigma_{\eta}$

Figure 13: Sensitivities $\frac{\partial I_{\eta}\left(\mathbf{x}_{0}, \mathbf{u}_{0}\right) / B_{\eta}(T)}{\partial \kappa_{\eta}}$ (a) and $\frac{\partial I_{\eta}\left(\mathbf{x}_{0}, \mathbf{u}_{0}\right) / B_{\eta}(T)}{\partial \sigma_{\eta}}$ as a function of $\kappa_{\eta}$ and $\sigma_{\eta}$.

For illustration, figure 13 depicts the isolines for sensitivity of $I_{\eta}\left(\mathbf{x}_{0}, \mathbf{u}_{0}\right) / B_{\eta}(T)$ to the absorption coefficient (figure 13a), and to the scattering coefficient (figure 13b), versus the absorption and scattering coefficients $\kappa_{\eta}$ and $\sigma_{\eta}$. The sensitivity to $\kappa_{\eta}$ decreases with the absorption coefficient, which means that the identification of $\kappa_{\eta}$ turns out to be more uncertain if the medium is optically thick. The sensitivity to $\sigma_{\eta}$ is much smaller than the sensitivity to $\kappa_{\eta}$ which suggests that the identification of $\sigma_{\eta}$ will be subject, in this case, to significant uncertainties.

The ability of the proposed SMC algorithm to deal with complex heterogeneous media is therefore very promising for high-temperature applications in gaseous or porous media. First of all, this technique can be used to get useful information about the feasibility of the radiative properties identification from measured radiative quantities. For instance, as shown in figure 13, the Jacobian matrix of sensitivities can be directly derived from the polynomials. If the identification of a parameter appears to be uncertain according to the analysis based on the SMC simulation, (such as the identification of $\sigma_{\eta}$ in the considered test case), two possibilities are offered:

- First, additional measurements of the radiative intensity (at other locations or/and directions) may provide better information about $\sigma_{\eta}$, and make the inverse pro- 
cedure feasible (as discussed in Sec 3.2, and illustrated in Fig 6). It is therefore possible to check in advance which measurements are the most relevant using the SMC simulations.

- Second, if others measurements are not available, the polynomial shows that the RTE may not be suitable for the identification. In this case, another model, which ensures that the inverse problem can be solved with a better accuracy, must be chosen. Only the RTE was considered as radiative transfer direct model in this work. Others models $[26,27]$ can be investigated if the identification procedure is not feasible with the RTE.

For situations where the inverse problem can be solved, the symbolic approach can improve significantly the efficiency of the inverse procedure. Indeed, only one single SMC run is needed to obtain the polynomial expansion. If an iterative procedure is considered, it is not necessary to solve the direct model at each iteration since the polynomial expansions can be directly used to estimate the radiative quantity considered. Therefore, the resolution of the RTE is needed only once. This feature of SMC is all the more exploitable so as the RTE resolution is more computationally demanding, such as in three-dimensional problems and/or in complex media.

All the Monte Carlo advances developed for radiative transfer, such as those reviewed in [22], benefits also to the SMC. Therefore, the symbolic approach proposed in this work can be extended without additionnal difficulties to more realistic problems (complex geometries, heterogeneous media with different structure, with reflection and refraction at the interface, etc.), in the same manner as for any standard MC methods.

However, as the number of unknown parameters increases, the symbolic Monte Carlo approach can become hardly tractable since it outputs high dimensional multivariate polynomials. In these cases, the symbolic approach proposed in this work may help to gather physical insight on some parameters. But, if reducing the dimension of the model (as it would decrease the number of unknown parameters) is not possible, SMC seems to be hardly recommendable for the identification.

\section{Conclusion}

The SMC approach described in this work allows for the first time expressing radiative quantities as polynomials of absorption and scattering coefficients, using the 
null-collision algorithm. All the radiative properties, including the absorption and scattering coefficients, can now be retained as symbolic variables without any knowledge of the optical thickness.

It opens new prospects for the identification of radiative properties of complex media since the SMC simulations appear to be very useful in various steps of the identification procedure. Firstly, they allow determining which measurements are more relevant for the considered problem. Secondly, they give significant information about the uniqueness of the solution. Thirdly, they can be directly used to develop efficient sensitivity analysis. This information is valuable to determine if the identification of the radiative properties is feasible. Moreover, the polynomials can also be used to improve the efficiency of an inverse method and decrease the computational cost. Indeed, these functional expressions may be used as a direct model in the inverse method, which avoid the costly resolution of the RTE at each iteration.

SMC benefits from all the advantages inherent in standard MC methods. Therefore it can be applied to complex three-dimensional problems. As shown in this work, problems such as the identification of radiative properties of two-phase media (such as porous materials), or the identification of temperature and absorption coefficients in combustion systems can be solved using the proposed technique. In future works, SMC will be applied to real porous media, and compared with experimental data.

Two numerical difficulties related to the use of polynomials can however be noticed. The first one is due to the so-called curse of dimensionality that occurs if the number of unknown parameters is important. Indeed, it becomes quickly difficult to deal with high dimensional multivariate polynomials. The second difficulty is related to cases where the order of polynomials becomes large, which occurs for large values of optical thicknesses. In these cases, machine precision issues can be encountered. This difficulty may be handled by mathematical developments on the polynomial forms. It will be the subject of future works.

\section{Appendix A. Symbolic standard deviation}

As for standard Monte Carlo algorithms, the standard deviation can be estimated with SMC (it is referred to as sample standard deviation [1]). This quantity - given here under a functional form - provides an estimate of the uncertainty in the estimated expected value. Using SMC, the radiative intensity is expressed as the expectation of a 
polynomial function and this appendix is dedicated to express standard deviations for such polynomials.

Let us first consider the case where a single variable $x$ is kept under a symbolic form, leading to a monovariate polynomial :

$$
p(x)=\sum_{n=0}^{\infty} a_{n} x^{n}
$$

where $a_{n}$ are random variables. The expected value of this random polynomial is approximated by SMC according to:

$$
E(p(x)) \approx \overline{p(x)}=\frac{1}{N} \sum_{i=1}^{N} \sum_{n=0}^{\infty} a_{i, n} x^{n}=\sum_{n=0}^{\infty} \overline{a_{n}} x^{n}
$$

where $N$ is the number of samples of the coefficients $a_{i, n}$ and $\overline{w_{n}}=\frac{1}{N} \sum_{i=1}^{N} w_{i, n}$.

The sample standard deviation is given by:

$$
s(\overline{p(x)})=\sqrt{\frac{N}{N-1}\left(\overline{p^{2}(x)}-\overline{p(x)}^{2}\right)}
$$

where $\overline{p^{2}(x)}$ is estimated by:

$$
\overline{p^{2}(x)} \approx \frac{1}{N} \sum_{i=1}^{N}\left(\sum_{n=0}^{\infty} a_{i, n} x^{n}\right)^{2}=\sum_{u=0}^{\infty} \sum_{v=0}^{\infty}\left(\sum_{i=1}^{N} \frac{1}{N} a_{i, u} a_{i, v} x^{u+v}\right)=\sum_{u=0}^{\infty} \sum_{v=0}^{\infty} \overline{a_{u} a_{v}} x^{u+v}
$$

and

$$
\overline{p(x)}^{2}=\left(\sum_{n=0}^{\infty} \overline{a_{n}} x^{n}\right)^{2}=\left(\sum_{u=0}^{\infty} \overline{a_{u}} x^{u}\right)\left(\sum_{v=0}^{\infty} \overline{a_{v}} x^{v}\right)=\sum_{u=0}^{\infty} \sum_{v=0}^{\infty} \overline{a_{u}} \overline{a_{v}} x^{u+v}
$$

with $\overline{a_{u} a_{v}}=\frac{1}{N} \sum_{i=1}^{N} a_{i, u} a_{i, v}, \overline{a_{u}}=\frac{1}{N} \sum_{i=1}^{N} a_{i, u}$ and $\overline{a_{v}}=\frac{1}{N} \sum_{i=1}^{N} a_{i, v}$.

The sample standard deviation $s(\overline{p(x)})$ can therefore be expressed as :

$$
s(\overline{p(x)})=\sqrt{\frac{N}{N-1} \sum_{u=0}^{\infty} \sum_{v=0}^{\infty}\left(\overline{a_{u} a_{v}}-\overline{a_{u}} \overline{a_{v}}\right) x^{u+v}}
$$

Following the same developments, the standard deviation can also be estimated in the case of a multivariate polynomial. For instance, considering the bivariate polynomial (i.e. characteristic of test cases where two parameters are unknown):

$$
p(x, y)=\sum_{m=0}^{\infty} \sum_{n=0}^{\infty} a_{m, n} x^{m} y^{n}
$$

$s(\overline{p(x, y)})$ is given by :

$$
s(\overline{p(x, y)})=\sqrt{\frac{N}{N-1} \sum_{m=0}^{\infty} \sum_{n=0}^{\infty} \sum_{m^{\prime}=0}^{\infty} \sum_{n^{\prime}=0}^{\infty}\left(\overline{a_{m, n} a_{m^{\prime}, n^{\prime}}}-\overline{a_{m, n}} \overline{a_{m^{\prime}, n^{\prime}}}\right) x^{m+m^{\prime} y^{n+n^{\prime}}}}
$$


with $\overline{a_{m, n} a_{m^{\prime}, n^{\prime}}}=\frac{1}{N} \sum_{i=1}^{N} a_{i, m, n} a_{i, m^{\prime}, n^{\prime}}, \overline{a_{m, n}}=\frac{1}{N} \sum_{i=1}^{N} a_{i, m, n}$ and $\overline{a_{m^{\prime}, n^{\prime}}}=\frac{1}{N} \sum_{i=1}^{N} a_{i, m^{\prime}, n^{\prime}}$.

Under pure statistical considerations, such functional expressions of standard deviations present a valuable advantage: the evolution of the uncertainty with the considered parameters is explicit, and may provide relevant information on the definition of arbitrary probabilities, as in importance sampling techniques (see Appendix B).

\section{Appendix B. Consequences of the arbitrary choice of scattering probability}

In the algorithm described in $\operatorname{Sec} 4.1$, the scattering coefficient $\sigma_{\eta}$ is unknown and arbitrary probabilities of scattering $\tilde{P}_{s}$ and null-collision $\tilde{P}_{n}=1-\tilde{P}_{s}$ are required. Even if the definition of such probabilities does not create any bias (an infinity of Monte Carlo samples would lead to the exact solution), some convergence issues can be encountered according to the given value of these probabilities. To be consistent with the statistics of the considered physics, $\tilde{P}_{s}$ and $\tilde{P}_{n}$ should be close to the ratios of the number of scattering events or null-collision events over the whole number of collisions:

$$
\tilde{P}_{s} \approx \frac{\sigma_{\eta}}{\sigma_{\eta}+\gamma_{\eta}}=\frac{\sigma_{\eta}}{\hat{\beta}_{\eta}-\kappa_{\eta}}
$$

and

$$
\tilde{P}_{n} \approx \frac{\gamma_{\eta}}{\sigma_{\eta}+\gamma_{\eta}}=\frac{\gamma_{\eta}}{\hat{\beta}_{\eta}-\kappa_{\eta}}
$$

However in SMC, the scattering coefficient is not defined and is considered as a variable. Therefore, a choice has to be done to define $\tilde{P}_{s}$ and $\tilde{P}_{n}$.

The influence of this choice is illustrated in Figs. B.14 where comparisons with standard Monte Carlo calculations are provided for a scattering, homogeneous and isothermal 1D medium of width $L$. The modified extinction optical thickness is fixed to $\hat{\tau}_{\eta}=\hat{\beta}_{\eta} L=5$ while the absorption optical thickness is $\tau_{a, \eta}=\kappa_{\eta} L=1$. A HenyeyGreenstein phase function with an asymmetry factor $g=0.3$ is considered. In those figures, the intensity $I_{\eta}\left(\mathbf{x}_{0}, \mathbf{u}_{0}\right) / B(T)$ is displayed as a function of the scattering optical thickness $\tau_{s, \eta}=\sigma_{\eta} L$ for different scattering probabilities $\tilde{P}_{s}$. The functional standard deviations (considered as confidence intervals) are also depicted in each figure.

In Fig. B.14a the scattering probability is set as $\tilde{P}_{s}=0.1\left(\tilde{P}_{n}=0.9\right)$. According to Eqs. B.1 and B.2, $\tilde{P}_{s}$ should be consistent with scattering optical thicknesses close to 0.4. As highlighted in this figure the polynomial remains accurate (by comparison with the standard Monte Carlo estimations) until $\tau_{s, \eta}=2$, and lose accuracy beyond this 


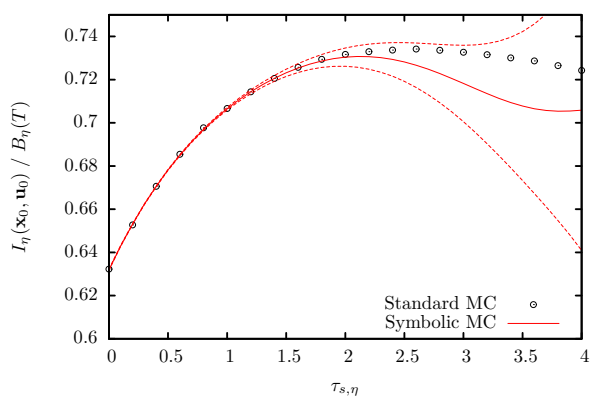

(a) $\tilde{P}_{s}=0.1$

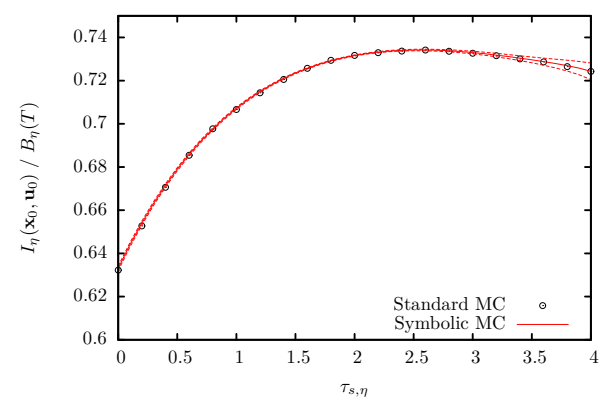

(b) $\tilde{P}_{s}=0.5$

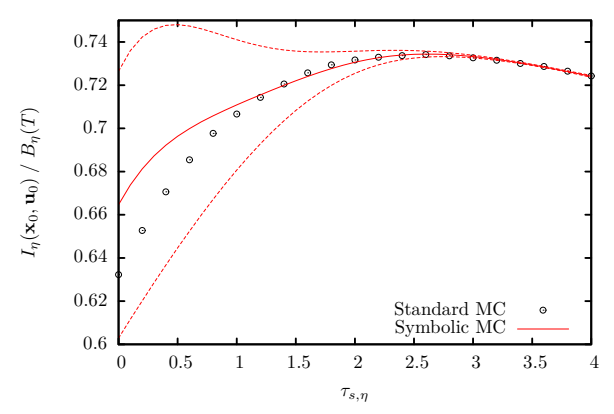

(c) $\tilde{P}_{s}=0.9$

Figure B.14: $I_{\eta}\left(\mathbf{x}_{0}, \mathbf{u}_{0}\right) / B_{\eta}(T)$ versus $\tau_{s, \eta}$ obtained with SMC with $\hat{\tau}_{\eta}=5, \tau_{a, \eta}=1$ and $N=10^{6}$ for three different scattering probabilities : $\hat{P}_{s}=0.1,0.5$ and 0.9 . Reference results estimated by standard Monte Carlo method are also displayed. 
value as far as $\tau_{s, \eta}$ increases. Indeed, the number of scattering events is underestimated for high values of $\tau_{s, \eta}$ and much more samples would be required to get accurate estimates. The confidence intervals are minimal for $\tau_{s, \eta}=0.4$ and significantly increase for $\sigma_{\eta}>2$ which confirms the comparisons with standard MC. Such a behavior indicates that the choice of $\tilde{P}_{s}=0.1$ (and $\tilde{P}_{n}=0.9$ ) could lead to convergence issues for the identification of large values of $\tau_{s, \eta}$. In the case where $\tilde{P}_{s}=\tilde{P}_{n}=0.5$, displayed in figure B.14b, the polynomial remains accurate for all $\tau_{s, \eta} \in[0,4]$ considered. Even if this choice of probability is relevant for $\tau_{s, \eta}$ values close to 2 (see Eqs. B.1 and B.2), the confidence interval remains small for the whole range of $\tau_{s, \eta}$ : no important underestimation or overestimation of scattering events is done. In the third case displayed in figure B.14c, the probability of scattering is set as $\tilde{P}_{s}=0.9\left(\tilde{P}_{n}=0.1\right)$. These probabilities are a good choice when $\tau_{s, \eta}$ is around 3.6. Under this value, the number of scattering events is overestimated, and an increase of confidence intervals is noticed, leading to wrong estimations of radiative intensities for small $\tau_{s, \eta}$.

For all cases described in this article, scattering probability about 0.5 have led to satisfying convergence behaviors. Nevertheless, good convergences are not guaranteed with this probability and we suggest to systematically compute the functional standard deviations in order to prevent some pathological behavior such as those discussed in Chapter 4, Sec. 4.2.1 of [1] or in Sec. 6 of [20].

[1] Dunn, W., Shultis, J.. Exploring Monte Carlo methods. Academic Press; 2012.

[2] Dunn, W.. Inverse Monte Carlo analysis. J Comput Phys 1981;41:154-166.

[3] Floyd, C., Jaszczak, R., Greer, K., Coleman, R.. Inverse Monte Carlo as a unified reconstruction algorithm for ECT. J Nucl Med 1986;27(10):1577-1585.

[4] Dunn, W.. Applications of the inverse Monte Carlo method in photon beam physics. Nucl Instrum Meth A 1987;255:147-151.

[5] Dunn, W.. Inverse Monte Carlo solution for radiative transfer in inhomogeneous media. J Quant Spectrosc Radiat Transf 1983;29:19-26.

[6] Subramaniam, S., Mengüç, M.. Solution of the inverse radiation problem for inhomogeneous and anisotropically scattering media using a Monte Carlo technique. Int J Heat Mass Transfer 1991;34(1):253-266. 
[7] Kaipio, J., Somersalo, E.. Statistical and Computational Inverse Problems. Springer; 2005.

[8] Howell, J., Siegel, R., Menguç, M.. Thermal Radiation Heat Transfer. Fifth Edition. CRC Press; 2011.

[9] Tarvainen, T., Kolehmainen, V., Arridge, S., Kaipio, J.. Image reconstruction in diffuse optical tomography using the coupled radiative transport-diffusion model. J Quant Spectrosc Radiat Transf 2011;112:2600-2608.

[10] Ren, T., Modest, M., Fateev, A., Clausen, S.. An inverse radiation model for optical determination of temperature and species concentration: Development and validation. J Quant Spectrosc Radiat Transf 2015;151:198-209.

[11] Sacadura, J.F.. Thermal radiative properties of complex media: Theoretical prediction versus experimental identification. Heat Transf Engineering 2011;32(9):754-770.

[12] Nicolau, V., Raynaud, M., Sacadura, J.. Spectral radiative properties identification of fiber insulating materials. Int J Heat Mass Transf 1994;37:311-324.

[13] Nisipeanu, E., Jones, P.. Identification of the effective radiative properties of a hot, thick, porous medium. J Quant Spectrosc Radiat Transf 1998;60(1):85-96.

[14] Milandri, A., Asllanaj, F., Jeandel, G.. Determination of radiative properties of fibrous media by an inverse method - comparison with the mie theory. J Quant Spectrosc Radiat Transf 2002;74:637-653.

[15] Kowsary, F., Gholamian, H., Ashjaee, M., Moghadassian, B.. Inverse identification of radiative properties of a multi-component media in mpa modeling of radiative transfer. Int J Communications Heat Mass Transf 2016;72:95-105.

[16] Baillis, D., Arduini-Schuster, M., Sacadura, J.. Identification of spectral radiative properties of plyurethane foam from hemispherical and bi-directional transmittance and reflectance measurements. J Quant Spectrosc Radiat Transf 2002;73:297-306.

[17] Patel, V., Talukdar, P.. Evaluation of radiative properties of a representative foam structure using blocked-off region approach integrated with finite volume method. Int J Thermal Sci 2016;108:89-99. 
[18] Galtier, M., Blanco, S., Caliot, C., Coustet, C., Dauchet, J., El Hafi, M., et al. Integral formulation of null-collision Monte Carlo algorithms. J Quant Spectrosc Radiat Transf 2013;125:57-68.

[19] Eymet, V., Poitou, D., Galtier, M., El Hafi, M., Terrée, G., Fournier, R.. Null-collision meshless Monte Carlo-Application to the validation of fast radiative transfer solvers embedded in combustion simulators. J Quant Spectrosc Radiat Transf 2013;129:145-157.

[20] Galtier, M., Blanco, S., Dauchet, J., El Hafi, M., Eymet, V., Fournier, R., et al. Radiative transfer and spectroscopic databases: A line-sampling Monte Carlo approach. J Quant Spectrosc Radiat Transf 2016;172:82-97.

[21] Howell, J.. The Monte Carlo method in radiative heat transfer. J Heat Transfer 1998;120(3):547-560.

[22] Delatorre, J., et al., . Monte Carlo advances and concentrated solar applications. Solar Energy 2014;103:653-681.

[23] N. Shamsundar E.M. Sparrow, R.H.. Monte Carlo radiation solutions-effect of energy partitioning and number of rays. Int J Heat Mass Transf 1973;16:690-694.

[24] Taine, J., Bellet, F., Leroy, V., Iacona, E.. Generalized radiative transfer equation for porous medium upscaling: Application to the radiative fourier law. Int J Heat Mass Transf 2010;53:4071-4081.

[25] Petrasch, J., Haussener, S., Lipiński, W.. Discrete vs. continuum-scale simulation of radiative transfer in semitransparent two-phase media. J Quant Spectrosc Radiat Transf 2011;112:1450-1459.

[26] Roger, M., Caliot, C., Crouseilles, N., Coelho, P.. A hybrid transport-diffusion model for radiative transfer in absorbing and scattering media. J Comput Phys 2014;275:346-362.

[27] Coelho, P., Crouseilles, N., Pereira, P., Roger, M.. Multi-scale methods for the solution of the radiative transfer equation. J Quant Spectrosc Radiat Transf 2016;172:36-49. 